\title{
Isolator-free, widely tunable thulium/holmium fiber laser
}

\author{
Anir Syazwan Sharbirin*, Mohammad Faizal Ismail, Harith Ahmad \\ Photonics Research Center, University of Malaya, 50603 Kuala Lumpur, Malaysia \\ * Corresponding author: asyazwan8@siswa.um.edu.my
}

\section{Article history}

Received 3 May 2018

Revised 25 August 2018

Accepted 11 September 2018

Published Online 25 October 2018

\section{Graphical abstract}

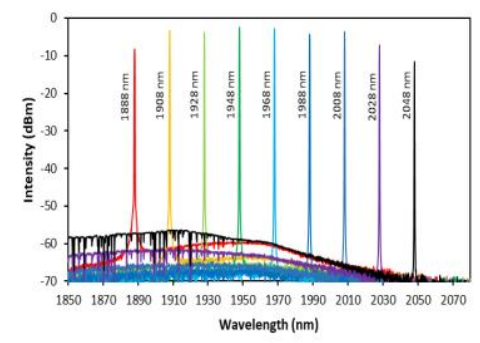

\begin{abstract}
An isolator-free thulium/holmium-doped fiber laser with a broadly tunable wavelength output is proposed and demonstrated for the first time. A theta resonator configuration is implemented in order to produce rectification of lasing direction without the need for an optical isolator, thus, making it a more cost-effective setup in comparison to the conventional ring resonator. Over $160 \mathrm{~nm}$ of wavelength tunability can be generated, which covers a huge range of the two-micron region starting from 1888 $\mathrm{nm}$ up to a maximum of $2048 \mathrm{~nm}$. The laser exhibits excellent wavelength control with its short-range wavelength tuning capability, whereby the shortest tuning spacing obtainable is as small as $\sim 0.1 \mathrm{~nm}$. The tunable laser peaks maintain a strong optical-signal-to-noise (OSNR) value for the whole tuning range, reaching more than $60 \mathrm{~dB}$, also a full-width half-maximum (FWHM) value less than $\sim 0.2 \mathrm{~nm}$ with a maximum output power of $6.82 \mathrm{~mW}$. The isolator-free cavity indicates a significant improvement in the slope efficiency of the laser in comparison to a ring cavity setup with similar components. The proposed laser would have substantial use as a laser seed for application in sensing and spectroscopy.
\end{abstract}

Keywords: Tunable fiber laser, thulium, holmium, theta cavity, isolator-free

(C) 2018 Penerbit UTM Press. All rights reserved

\section{INTRODUCTION}

In recent years, research in fiber lasers has shifted its focus from the common 1530 - $1565 \mathrm{~nm}$ band by the Erbium-doped Fiber Amplifier (EDFA) to develop other noteworthy wavelength bands such as the two-micron region. This spectral band which is at the range of $1700-2400 \mathrm{~nm}$ is associated with the Thulium-doped Fiber Amplifier (TDFA), which has been progressively studied to be used for future optical communications $[1,2]$. Nonetheless, the true attraction of this unique spectral band is that it is enriched with strong absorption lines for water molecules and atmospheric gases $[3,4]$. This characteristic of the wavelength band allows it to be utilized for a multitude of applications in sensing [5, 9-11] and spectroscopy [12-14], especially by configuring it into a tunable two-micron laser source [5-8].

Generally, a fiber ring cavity is the preferred configuration for rareearth-doped fiber lasers $[9,10]$. This type of cavity is essentially a ring resonator which employs an optical isolator to ensure unidirectional propagation of light in order to generate single-frequency lasing operation. However, a $\sim 2 \mu \mathrm{m}$ optical isolators is an expensive component due to the eccentricity of the two-micron wavelength band in comparison to the conventional telecommunication band. The optical isolators also operate in a narrow operating bandwidth, usually around tens of nanometers. Thus, optical isolators may not be the best choice for wide-tuning operations [11].

Quite recently, a paper by Kharitonov et. al have proposed an unconventional isolator-free theta cavity for two-micron lasers [11-13] The theta resonator is essentially a combination of two ring resonators which will fundamentally suppress a certain propagation direction. In other words, this cavity does not require an optical isolator for rectifying the lasing path thus, providing a cost-effective and reliable solution to generate a widely tunable dual-wavelength two-micron fiber laser $[11,12,14]$.
This work proposes and demonstrates an isolator-free, widely tunable two-micron fiber laser. The proposed laser uses a theta resonator configuration with a pair of 2 X 2, 3-dB couplers to enable an $\mathrm{S}$-shaped feedback. In order to achieve a tunable laser emission within the two-micron wavelength range, a tunable bandpass filter is employed as the tuning agent together with a Thulium/Holmium-doped fiber (THDF) as the preferred gain medium. THDF is principally a co-doped fiber which exploits the pump absorption of $\mathrm{Tm}^{3+}$ ions at $1.55 \mu \mathrm{m}$ to generate light at $\sim 1.8-2.0 \mu \mathrm{m}$. The emitted light will consequently excite the $\mathrm{Ho}^{3+}$ ions at ${ }^{5} \mathrm{I}_{7} \rightarrow{ }^{5} \mathrm{I}_{8}$ and allow for emission at $1.95-2.1 \mu \mathrm{m}$ $[3,15]$. To the best of the author's knowledge, this is the first demonstration of a tunable wavelength Thulium/Holmium-doped Fiber Laser (THDFL) implementing a theta resonator configuration. The proposed laser has a promising use in the application of sensing and spectroscopy.

\section{FUNDAMENTAL PRINCIPLES OF THETA RESONATOR}

The theta cavity provides rectification of lasing direction by introducing non-reciprocal cavity loss in such a way that it completely suppresses light propagation at a specific direction. The non-reciprocal signal loss is achieved by constructing an S-shaped feedback within a ring cavity, creating a theta-like pattern. Fig. 1 describes a simple model of the theta resonator [11]. The model considers a ring resonator with a lumped gain $G$ and loss $L$ with two directional couplers (DC1 and DC2) creating an S-shaped feedback which ultimately dictates the behavior of the light propagation within the cavity. 


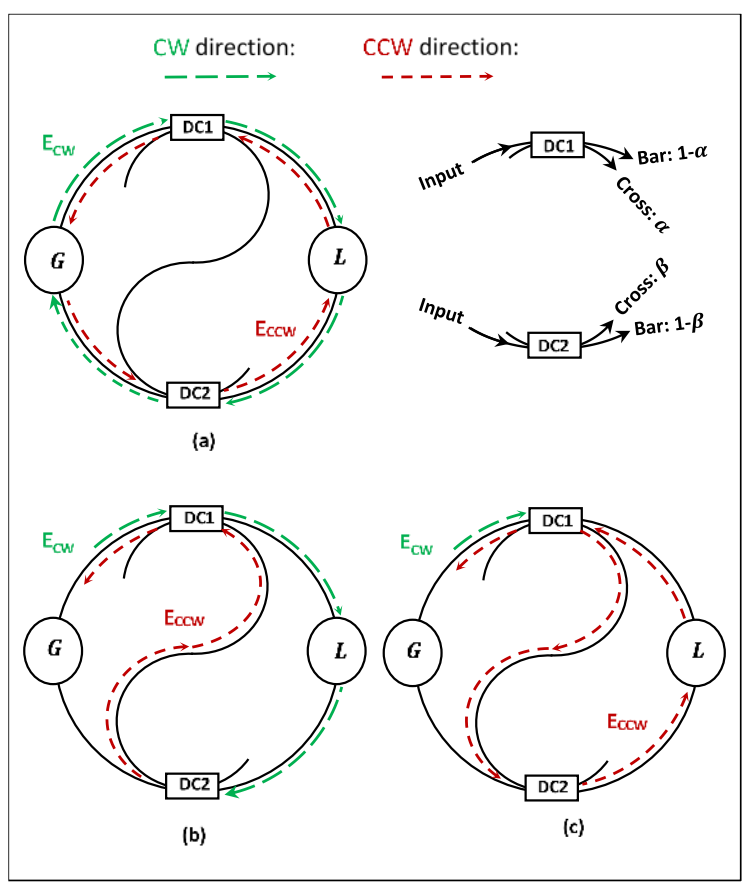

Fig. 1 Basic model of theta resonator (a) the main CW and CCW paths. (b) First possible rectifying signal from $\mathrm{CW}$ to CCW. (c) Second possible rectifying path from $\mathrm{CW}$.

The light signal propagates in two directions; clockwise and

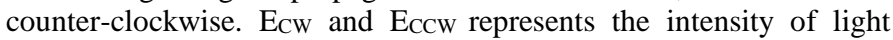
propagating in each direction. The cross-coupling ratio of DC1 and DC2 are depicted as $\alpha$ and $\beta$ respectively, meanwhile $(1-\alpha)$ and $(1-$ $\beta$ ) are terms referring to the coupling output into the main ring path. These terms are further illustrated as in Fig. 1. Ecw and Eccw mainly circulate along the outer ring as in Fig. 1 (a). However, the connected cross output of DC1 and DC2 reroutes the $E_{C w}$ to the $E_{C C W}$ path as shown in (Fig. 1 (b) and (c)). The $\mathrm{n}^{\text {th }}+1$ round trip for Eccw and Ecw based on Fig. 1 can be expressed by Equations (1) and (2) respectively:

$E_{C C W, n} G_{C C W}(1-\alpha) L(1-\beta)+E_{C W, n}, G_{C W}(1-\beta) \alpha \beta L+$

$E_{C W, n} G_{C W} \alpha \beta(1-\beta) L=E_{C C W, n+1}$

$E_{C W, n} G_{C W}(1-\alpha) L(1-\beta)=E_{C W, n+1}$

$G_{C W}$ and $G_{C C W}$ represents the gain acquired by the light $\left(E_{C W}\right.$ and $\left.E_{C C W}\right)$ as it passes by the clockwise or anticlockwise direction. The first term for Equation (1) is the main path for $E_{C C W}$ as in Fig. 1 (a), while the second and the third term describes the conversion of the $E_{C W}$ to the $E_{C C W}$ path as in Fig. 1 (b) and (c). Meanwhile, Equation (2) describes the main path for $E_{C W}$ as described in Fig. 1(a). A glance at Equation (1) and (2) foreshadows that $E_{C C W}$ will repress $E_{C W}$ since a significant portion of $E_{C W}$ diverts to the $E_{C C W}$ path and not otherwise.

In a steady state regime, $E_{C W, n+1}=E_{C W, n}=E_{C W}$ and similarly to $E_{C C W}$. We can then rewrite (2) as:

$[(1-\alpha)(1-\beta) L)]^{-1}=G_{C W}$

Then, by substituting (3) into (1), the following expression is obtained:

$E_{C C W} \frac{G_{C C W}}{G_{C W}}+2 E_{C W} \frac{\alpha \beta}{1-\beta}=E_{C C W}$

By assuming steady-state condition, $G_{C C W}=G_{C W}$, hence, canceling out the $E_{C C W}$ part in (4), which will also lead to $E_{C W}=0$. In other words, the CCW signal prevails over the $\mathrm{CW}$ component. Based on (4), since $E_{C W}=0$, this means that at the steady-state condition, the CW signal will always be suppressed regardless of the coupling ratio.
However, based on previous investigation, this is not the case as the coupling ratio will affect the performance of the laser $[11,16]$.

\section{EXPERIMENTAL SETUP}

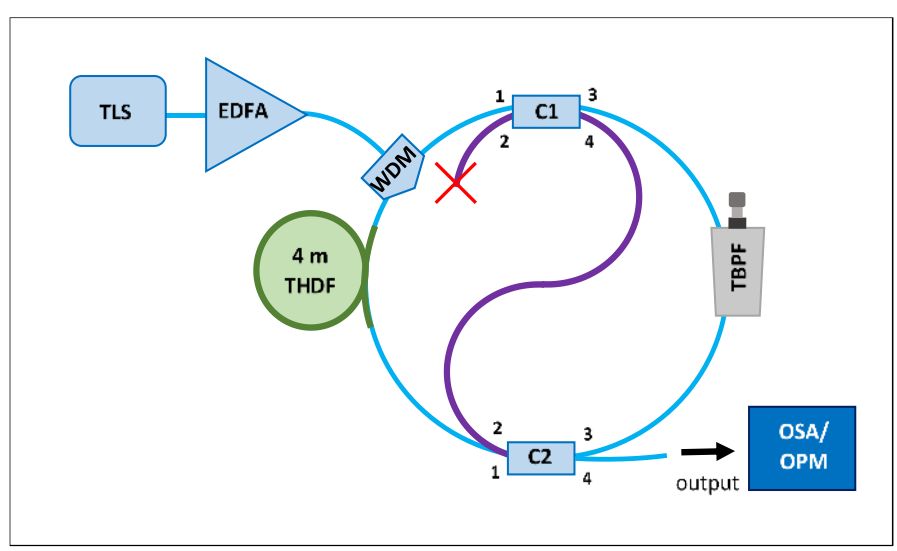

Fig. 2 Isolator free tunable THDFL theta cavity setup.

A wavelength-tunable THDFL operating in the two-micron region using a theta resonator configuration is shown in Fig. 2. The THDFL cavity utilizes a single-mode Coractive TH512 Thulium/Holmiumdoped Fiber (THDF) as the gain medium to provide emission within the two-micron wavelength band. The gain medium is a 4-meter-long fiber with a numerical aperture (NA) of 0.16 and a core absorption of $\sim 21$ $\mathrm{dBm}$ at $1.55 \mu \mathrm{m}$. The laser pump for the cavity comprises of a C-band tunable light source (TLS) that is set at a center wavelength of $1.55 \mu \mathrm{m}$ which is then amplified using a Keopsys Erbium-doped Fiber Amplifier (EDFA) capable of reaching a high output power within the range of watts. The pump is integrated into the cavity by using a $1550 / 2050 \mathrm{~nm}$ Haphit wavelength division multiplexer (WDM). Wide wavelength tunability is achieved by employing a tunable bandpass filter (TBPF) from Agiltron which functions as the wavelength-selective feedback mechanism. The TBPF is a compact and rugged device that filters out specific wavelength in order to allow excellent wavelength tuning in the two-micron region.

The rectification of feedback signal is done with the absence of optical isolators. This is executed by deploying a pair of 3-dB $2 \times 2$ couplers $(\mathrm{C} 1$ and $\mathrm{C} 2)$, whereby their respective cross-outputs are connected to form an S-shaped feedback. The S-shaped feedback forms the theta-like shape which introduces non-reciprocal losses in order to achieve light rectification. $\mathrm{C} 1$ and $\mathrm{C} 2$ are connected via their crossoutputs that is port 4 and port 2 respectively. Port 2 of $\mathrm{C} 1$ is unused, thus, it is terminated. $\mathrm{C} 1$ is placed inside the cavity by connecting the port 1 to the $2000 \mathrm{~nm}$ port of the WDM and port 3 to the TBPF. On the other hand, $\mathrm{C} 2$ is placed between the THDF and TBPF that is respectively connected to port 1 and port 3 of $\mathrm{C} 2$. Finally, the output of the laser is taken from port 4 of $\mathrm{C} 2$ which is taken for the measurement of the wavelength spectrum by using a Yokogawa AQ6375 Optical Spectrum Analyzer (OSA) and the average output power is by using a Thorlabs S302C optical power meter (OPM).

\section{RESULTS AND DISCUSSION}

A Thulium/Holmium-doped fiber laser (THDFL) working in the two-micron region is generated by employing the configuration as proposed in Fig. 2. Fig. 3 describes the single lasing operation with a center wavelength $\lambda_{c}$ of $1968 \mathrm{~nm}$ that is generated at a threshold of 532 $\mathrm{mW}$ with an output power of $2.71 \mathrm{~mW}$. The laser itself has a strong optical-signal-to-noise ratio (OSNR) of $\sim 65 \mathrm{~dB}$ with a full-width halfmaximum (FWHM) of $\sim 0.18 \mathrm{~nm}$. By adjusting the tuning knob of the TBPF, wide wavelength tunability is achieved from $1888 \mathrm{~nm}$ to 2048 $\mathrm{nm}$, with $20 \mathrm{~nm}$ spacing giving a total of $160 \mathrm{~nm}$ of tuning range as described in Fig. 4. The whole lasing spectrum maintains an OSNR above $\sim 60 \mathrm{~dB}$ with an average FWHM of less than $\sim 0.2 \mathrm{~nm}$. 


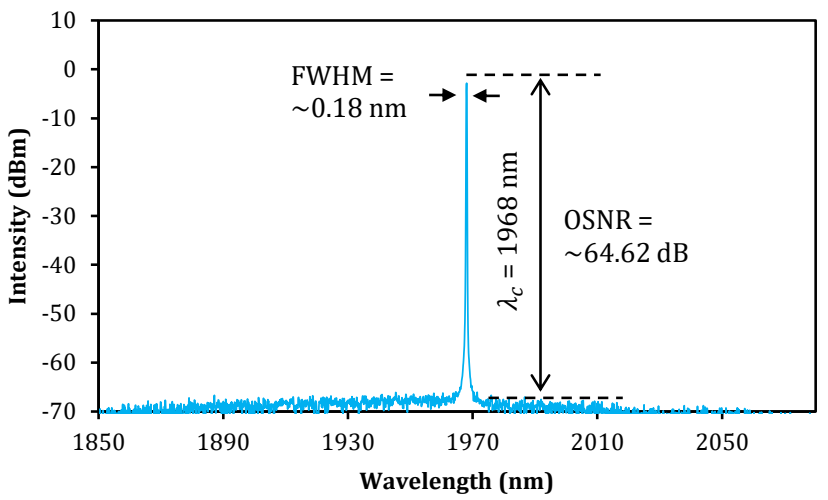

Fig. 3 Laser emission at $1968 \mathrm{~nm}$ from the THDFL theta cavity setup.

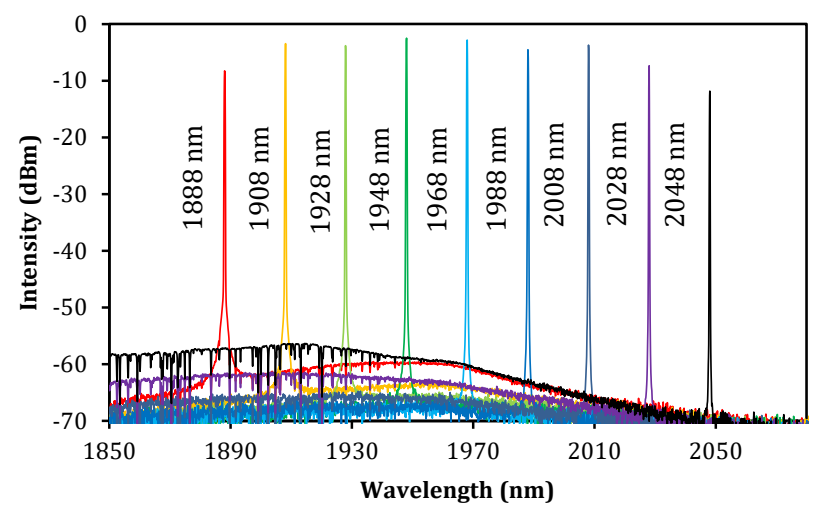

Fig. 4 Full tuning spectrum of the THDFL from $1888 \mathrm{~nm}$ to $2048 \mathrm{~nm}$.

In order to test the two-micron filtering reliability of the TBPF, a wavelength stability test is done. The optical trace of the laser for each wavelength as in Fig. 4 is taken at ten minute interval for a total of onehour operation. Then, the respective center wavelength of each laser trace is measured and plotted as in Fig. 5. The configured laser shows excellent wavelength stability whereby no mode-hopping can be observed for the whole one-hour operation. Additionally, the laser shows minor wavelength drift at an average of $\sim 0.03 \mathrm{~nm}$ from the center wavelength.

The tunable THDFL setup also demonstrates considerably good wavelength-control as it is capable to perform short wavelength-tuning with spacing as small as $\sim 0.1 \mathrm{~nm}$ as described in Fig. 6. Finally, the isolator-free, theta cavity THDFL setup is compared with a conventional ring cavity laser. A straightforward ring cavity is constructed whereby the coupler $\mathrm{C} 1$ in Fig. 2 is replaced with a 2050 $\mathrm{nm}$ isolator. Since both laser setup utilizes a common TBPF, the laser output should have similar wavelength tunability, OSNR, and linewidth. Therefore, only the output power to the input power slope efficiency of the setup is compared. The comparison wavelength chosen is at $1968 \mathrm{~nm}$ output which is at the center of the wavelength tuning spectrum as in Fig. 4. Fig. 7 shows that the efficiency of the proposed theta cavity design significantly outperforms the conventional ring design. The optical isolator works by allowing unidirectional transmission of light within the fiber by Faraday effect. However, the optical isolator suffers from having a small operating bandwidth. In this work, the only available optical isolator at hand has an operating wavelength of $2050 \pm 20 \mathrm{~nm}$. At $1968 \mathrm{~nm}$, the optical isolator in use is inherently unsuitable for the lasing wavelength, thus, explaining the huge difference between the efficiency of the lasers in study. If the operating bandwidth of the isolator matches the laser wavelength in study, the characteristics of the laser design should be similar as previously investigated by Kharitonov et. al [11].Therefore, this shows that in the absence of a suitable optical isolator, the theta cavity design is a much better alternative.
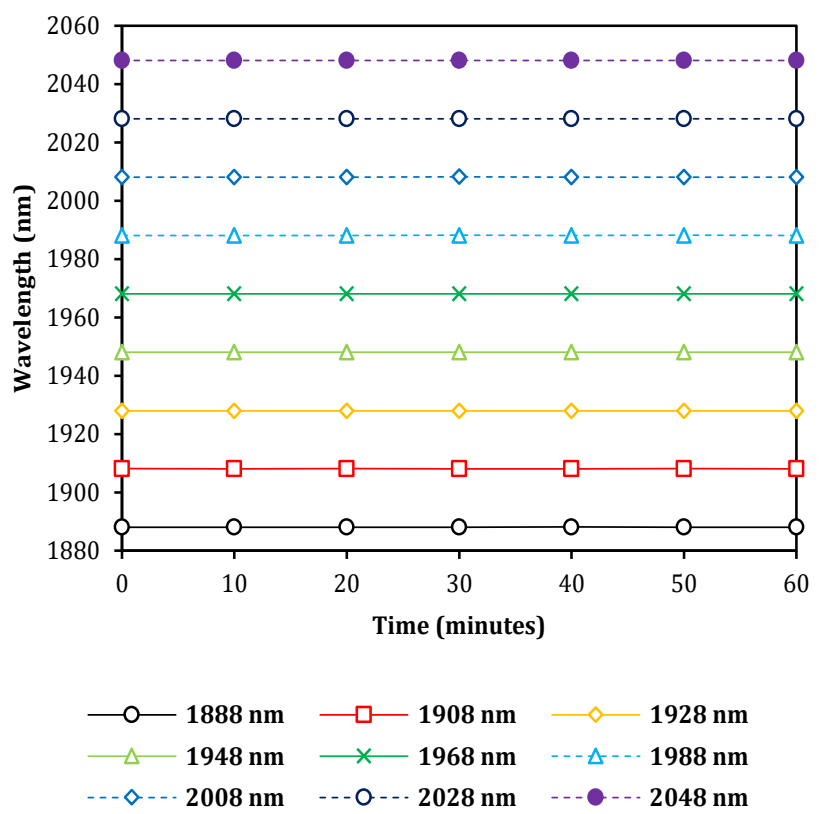

Fig. 5 Wavelength stability of the THDFL tested for one-hour operation.

Fig. 6 Small tuning capability of the THDFL.

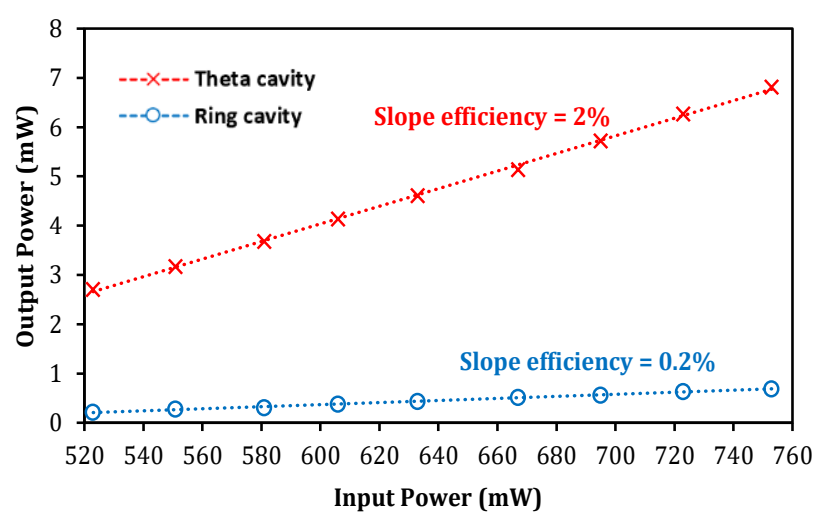

Fig. 7 Comparison of the slope efficiency between the theta cavity and the ring cavity design.

It is also noted from previous literature that the efficiency of the laser varies with the chosen cross-coupling ratios [11,16]. In term of OSNR, FWHM and wavelength-tuning capability, the isolator-free cavity design is not underperformed in comparison to our previous work [9]. However, the efficiency of the laser could be improved provided that better optical components and SM 2000 fiber is used for the cavity design. Nevertheless, this tunable THDFL configuration has a cost-effective design with promising potential as a tunable laser seed especially for sensing application.

\section{CONCLUSION}

A tunable two-micron Thulium/Holmium-doped fiber laser implementing a theta cavity is proposed and demonstrated in this work. The theta cavity enables a cost-effective isolator-free design whereby the rectification of lasing direction is dictated by the $S$-shaped feedback of the couplers. The tunable wavelength signal is produced by making use of a TBPF which is capable of tuning capability up to $\sim 160 \mathrm{~nm}$ with controllable spacing as small as $\sim 0.1 \mathrm{~nm}$. The generated two-micron laser has excellent stability with an OSNR value of more than $\sim 60 \mathrm{~dB}$ and FWHM of less than $\sim 0.2 \mathrm{~nm}$. The proposed TDWFL has a significant potential to be used mainly for sensing applications. 


\section{REFERENCES}

[1] Li, Z., Heidt, A. M., Simakov, N., Jung, Y., Daniel, J. M. O., Alam, S. U., et al. (2013). Diode-pumped wideband thulium-doped fiber amplifiers for optical communications in the $1800-2050 \mathrm{~nm}$ window. Optics Express, 21(22), 26450-26455.

[2] Li, Z., Heidt, A., Daniel, J., Jung, Y., Alam, S., and Richardson, D. J. (2013). Thulium-doped fiber amplifier for optical communications at 2 um. Optics Express, 21(8), 9289-9297.

[3] Scholle, K., Lamrini, S., Koopmann, P., and Fuhrberg, P. (2010). $2 \mu \mathrm{m}$ Laser Sources and Their Possible Applications. In B. P. (Ed.) Frontiers in Guided Wave Optics and Optoelectronics (Vol. 1, pp. 471-500). Germany: InTech.

[4] Curcio, J. A., Petty, C. C. (1951). The near infrared absorption spectrum of liquid water. Journal of the Optical Society of America, 41(5), 302304.

[5] Ahmad, H., Sharbirin, A. S., Muhamad, A., Samion, M. Z., Ismail, M. F (2017). $2 \mu \mathrm{m}$ mode-locked thulium-doped fiber laser using MachZehnder interferometer tuning capability. Laser Physics, 27(6), 065104.

[6] Ghosh, A., Roy, A. S., Chowdhury, S. D., Sen, R., Pal, A. (2016). Allfiber tunable ring laser source near $2 \mu \mathrm{m}$ designed for $\mathrm{CO}_{2}$ sensing. Sensors and Actuators B: Chemical, 235, 547-553.

[7] Zheng, S., Zhu, Y., and Krishnaswamy, S. (2013). Tunable fiber ring laser absorption spectroscopic sensors for gas detection. Sensors and Smart Structures Technologies for Civil, Mechanical, and Aerospace Systems, 8692, 869213-869219.

[8] McAleavey, F. J., Gorman, J. O., Donegan, J. F., MacCraith, B. D., Hegarty, J., Mazé, G. (1997). Narrow linewidth, tunable $\mathrm{Tm}^{3+}$ - doped fluoride fiber laser for optical-based hydrocarbon gas sensing. Selected Topics in Quantum Electronics, IEEE Journal of selected topics in quantum electronics, 3(4), 1103-1111.

[9] Sharbirin, A. S., Samion, M. Z., Muhamad, A., Ismail, M. F., and Ahmad, H. (2017). Bidirectional-pumped tunable fiber laser using a voltagecontrolled Fabry-Perot Etalon filter. Malaysian Journal of Fundamental and Applied Sciences, 13(3), 290-293.

[10] Ahmad, H., Sharbirin, A. S., Samion, M. Z., and Ismail, M. F. (2017). All-fiber multimode interferometer for the generation of a switchable multi-wavelength thulium-doped fiber laser. Applied Optics, 56(21), 5865-5870.

[11] Kharitonov, S., Bres, C.-S. (2015). Isolator-free unidirectional thuliumdoped fiber laser. Light: Science \& Applications, 4(10), e340.

[12] Kharitonov, S., and Brès, C.-S. (2017). Dual-emission band all-fiber laser based on theta cavity with thulium-and holmium-doped fibers. Paper presented at the Optical Fiber Communications Conference and Exhibition (OFC), 19-23 March 2017, Los Angeles, CA, USA, 1-3.

[13] Kharitonov, S., Brès, C.-S. (2016). Unidirectional all-fiber thuliumdoped laser based on theta cavity and fiber Bragg grating as filtering element. Paper presented at the Lasers Congress 2016 (ASSL, LSC, LAC), 30 October 2016, Boston, Massachusetts, AM5A 5.

[14] Godard, A. (2007). Infrared $(2-12 \mu \mathrm{m})$ solid-state laser sources: A review. Comptes Rendus Physique, 8(10), 1100-1128.

[15] Chernysheva, M., Mou, C., Arif, R., AlAraimi, M., Rümmeli, M., Turitsyn, S., et al. (2016). High Power Q-Switched Thulium Doped Fibre Laser using Carbon Nanotube Polymer Composite Saturable Absorber. Scientific Reports, 6, 24220. 